\title{
The dynamics of the $\gamma$ Vel cluster and nearby Vela OB2 association
}

\author{
Joseph J. Armstrong, ${ }^{\star}$ Nicholas J. Wright, R. D. Jeffries and R. J. Jackson \\ Astrophysics Group, Keele University, Staffordshire ST5 5BG, UK
}

Accepted 2020 March 30. Received 2020 March 30; in original form 2020 January 3

\begin{abstract}
The kinematics of low-mass stars in nearby OB associations can provide clues about their origins and evolution. Combining the precise positions, proper motions, and parallaxes given in the second Gaia Data Release with radial-velocity measurements obtained with the Hermes spectrograph at the Anglo-Australian Telescope, we have an opportunity to study in detail the kinematics of low-mass stars belonging to the nearby $\gamma$ Vel cluster and the Vela OB2 association it is projected against. The presence of lithium is used to confirm the youth of our targets. We separate our sample into the cluster and association populations based on the Gaia-ESO Survey membership probabilities their parallaxes, and kinematics. We find strong evidence for expansion in the OB association population with at least $4 \sigma$ significance along all three axes, though the expansion is notably anisotropic. We discuss these results in the context of cluster and association dispersal theories.
\end{abstract}

Key words: methods: data analysis - techniques: photometric-surveys - open clusters and associations: individual: Vela OB2, $\gamma$ Velorum.

\section{INTRODUCTION}

OB associations are sparse groups of kinematically associated but gravitationally unbound stars. Their brightest members have been studied for many years (Ambartsumian 1947; Blaauw 1964), but it is only recently that low-mass stars belonging to these associations have begun to be identified over large areas (Preibisch et al. 2002; Briceño et al. 2007; Armstrong, Wright \& Jeffries 2018; CantatGaudin et al. 2019a).

One hypothesis explaining the origin of $\mathrm{OB}$ associations is that they are the unbound remnants of previously bound young clusters (Tutukov 1978; Hills 1980; Kroupa, Aarseth \& Hurley 2001). After a few Myrs, newly formed O- and B-type stars sweep away the molecular cloud in which the cluster is embedded via feedback from photoionizing radiation and stellar winds, and the loss of this binding mass causes the cluster to become unbound and disperse. However, more recent work using astrometric data from Gaia (Gaia Collaboration et al. 2016; Gaia Collaboration et al. 2018) has begun to challenge this hypothesis. Wright \& Mamajek (2018) analysed the dynamics of the Scorpius-Centaurus OB2 association and found that it did not display the radial expansion pattern expected if the association had been formed as a more compact cluster (or clusters). Rather, they concluded that the association was most likely formed in multiple highly substructured subgroups, a view supported by the age distribution found by Pecaut \& Mamajek (2016). Ward \& Kruijssen (2018) examined kinematic parameters of 18 nearby associations and concluded that none of these associations showed signs of evolving from clusters. This new evidence implies that star

\footnotetext{
^E-mail: j.armstrong@keele.ac.uk
}

formation can take place over regions of various densities and that regions of high local density form gravitationally bound clusters, while low-density regions form unbound associations (Kruijssen 2012). Kuhn et al. (2019) investigate the kinematics of 28 clusters and associations by looking at young stellar objects in Gaia DR2 and find that at least 75 percent show signatures of expansion with a median velocity of $\sim 0.5 \mathrm{~km} \mathrm{~s}^{-1}$. Their results indicate that some young clusters can contain significant substructure and do still exhibit the potential to expand to the scales of $\mathrm{OB}$ associations.

The $\gamma$ Velorum cluster is a dense group of young stars (1020 Myr; Pozzo et al. 2000; Jeffries et al. 2009, 2017) located in the Vela OB2 complex (at a distance of $\sim 410 \mathrm{pc}$; de Zeeuw et al. 1999), which includes many lithium-rich, pre-main sequence (PMS) stars. The study by Jeffries et al. (2014) using radial velocities (RVs) from the Gaia-ESO Survey (GES; Gilmore et al. 2012; Randich, Gilmore \& Gaia-ESO Consortium 2013) identified two kinematically distinct populations within their sample of stars towards the cluster, population $\mathrm{A}$, a compact component with a narrow 1D RV dispersion $\sigma_{\mathrm{A}}=0.34 \pm 0.16 \mathrm{~km} \mathrm{~s}^{-1}$ and a potentially more widespread population $\mathrm{B}$ with a broader $\mathrm{RV}$ dispersion $\sigma_{\mathrm{B}}=$ $1.60 \pm 0.37 \mathrm{~km} \mathrm{~s}^{-1}$, whose mean RVs are offset by $2.15 \pm 0.48$ $\mathrm{km} \mathrm{s}^{-1}$. Sacco et al. (2015) established that the $\sim 35 \mathrm{Myr}$ cluster NGC 2547 , located $\sim 2$ degrees south of $\gamma$ Vel, also exhibits two kinematically distinct populations, and that populations NGC 2547 $\mathrm{B}$ and $\gamma$ Vel B have similar RVs and lithium abundances.

Since the release of Gaia data, the Vela OB2 region has been the subject of a number of studies focused on identifying and studying the young population across the association (Damiani et al. 2017; Armstrong et al. 2018; Beccari et al. 2018; Franciosini et al. 2018; Cantat-Gaudin et al. 2019b,a). Franciosini et al. (2018) focused on the $\gamma$ Vel cluster and found that populations A and B are 


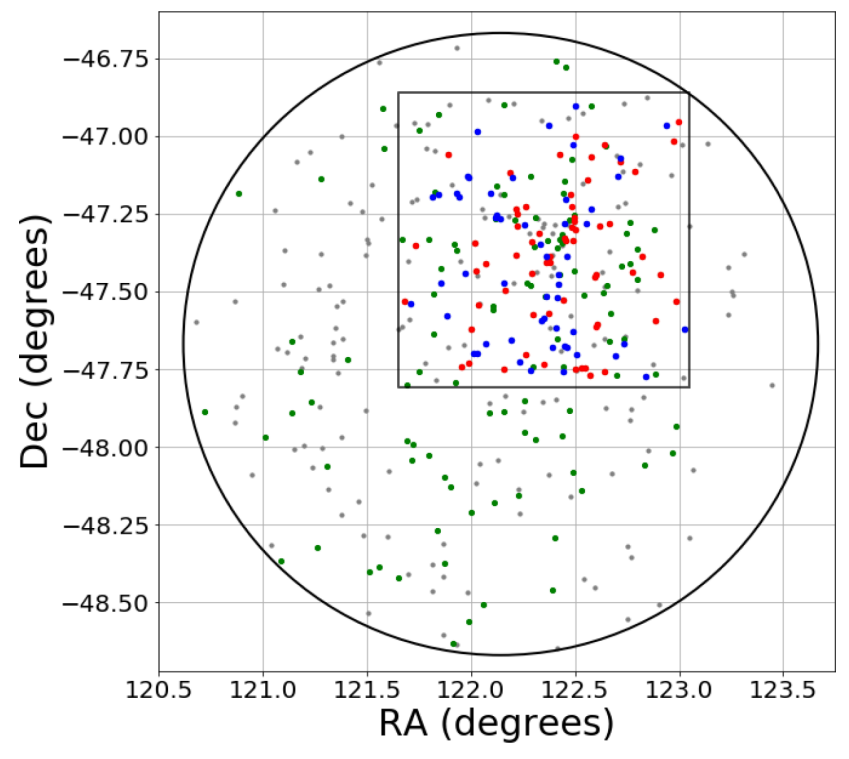

Figure 1. Positions of combined GES (square area) and AAT (AngloAustralian Telescope; circular area) sample sources. Sources with EW(Li) $>150 \mathrm{~m} \AA$ (green) along with population A (red) and B (blue) members with membership probabilities $>0.75$ from Jeffries et al. (2014).

separate in parallax as well as $\mathrm{RV}\left(\varpi_{\mathrm{A}}=2.85 \pm 0.008\right.$ mas, $\varpi_{\mathrm{B}}=$ $2.608 \pm 0.017$ mas), making $\gamma$ Vel A $\sim 38$ pc closer, and also found an inverse correlation between parallax and RV in $\gamma$ Vel B, which suggests that this group is expanding. Beccari et al. (2018) made use of the density-based spatial clustering of applications with noise (DBSCAN; Ester et al. 1996) clustering algorithm to identify six groups of stars within a $\sim 55 \mathrm{deg}^{2}$ area, four of which are distinct in position and proper motion space including both $\gamma$ Vel and NGC 2547. These clusters correlate with the extended PMS population of Vela OB2 identified by Armstrong et al. (2018) using Gaia DR1 and 2MASS photometry. Cantat-Gaudin et al. (2019b) combine Gaia DR2 astrometry and GES data and select stars using photometry and the unsupervized photometric membership assignment in stellar clusters (UPMASK; Krone-Martins \& Moitinho 2014) scheme to group stars based on their proper motions and parallax. Among stars of Vela OB2 identified, they find the distribution is fragmented into 11 components arranged in a ring-like structure around the IRAS Vela Shell. They consider the possibility that the expansion of the shell triggered star formation in Vela OB2. Cantat-Gaudin et al. (2019a) then use UPMASK to group stars in a large field across the Vela-Puppis region (>600 degrees). They identify seven distinct populations of various ages (ranging from $\sim 8$ to $50 \mathrm{Myr}$ ), all of which show signs of expansion in the Galactic $\mathrm{X}$ and $\mathrm{Z}$ dimensions and substructure in positions and kinematics.

In order to better understand the 3D dynamical state and evolution of the wider Vela OB2 association, we have undertaken a spectroscopic survey of the region. In this study, we present the first results from this survey for a 1 degree radius field covering the $\gamma$ Vel cluster and the existing GES field.

\section{DATA}

\subsection{Target selection}

We selected all Gaia DR1 sources within a 1 degree radius of (1, b) $=\left(263^{\circ},-8^{\circ}\right)$, which overlaps the GES $\gamma$ Vel field (see Fig. 1), within the Gaia-magnitude $(\mathrm{G})$ range 14.5-17.5 mag. These were then matched by position with the 2MASS catalogue (Skrutskie et al. 2006) with a matching radius of 0.5 arcsec. Sources with J, H, K photometry uncertainties $>0.05$ mag or with possible contamination (as indicated by the 'Cflg' flag) were excluded. We then filter these sources through a G-K versus G colour-magnitude selection and a $\mathrm{H}-\mathrm{K}$ versus J-H colour-colour selection using the method described in Armstrong et al. (2018), producing a sample of 360 likely PMS stars as targets for observation.

\subsection{Observations and data reduction}

Observations were made with the 2-degree field (2dF, Lewis et al. 2002) fibre positioner and the high-efficiency and resolution multi-element spectrograph (HERMES; Sheinis et al. 2015) at the Anglo-Australian Telescope (AAT) on 2018 February 6. HERMES observes in four optical bands and the red band within which the Li I $6708 \AA$ absorption line is found covers the wavelength range $647.8-673.7 \mathrm{~nm}$. This is divided among 4096 pixels $(0.0063 \mathrm{~nm}$ per pixel) on the CCD and yields a typical SNR of 100 per resolution element in $60 \mathrm{~min}$ for a source of magnitude $V=14$ (Sheinis et al. 2015). Three $2400 \mathrm{~s}$ exposures were completed for the field covering the $\gamma$ Vel cluster. Calibration frames, including wavelength calibration frames, dark frames, and multifibre flat fields, were taken with the exposures to be subtracted from the target spectra during reduction (Lewis et al. 2002). Also, 25 fibres per field were allocated to regions of empty sky in the field to measure the 'sky spectrum', which is then subtracted from the target spectra. The spectroscopic data were reduced using the software 2DFDR (AAO Software Team 2015). RVs were measured from the reduced spectra by cross-correlating the median spectra of individual targets with the spectra of standard stars and then fitting a Gaussian function to characterize the peak in the cross-correlation function, following the procedure of Jackson \& Jeffries (2010) and Jackson, Deliyannis \& Jeffries (2018). We match our AAT targets by position with the Vista Hemisphere Survey catalogue (VHS; McMahon et al. 2013) and combine the VHS and 2MASS $K$-band measures by taking the 2 MASS value for $K<12$, taking the mean of the two measures for $12<K<13$, and then using VHS for $K>13$. We then use Gaia DR2 G magnitude to calculate the G-K colour for these sources and perform spectral energy distribution (SED) fitting using the method outlined in Wright et al. (2019) to estimate effective temperatures $\left(T_{\text {eff }}\right)$.

In order to determine equivalent widths (EWs) for the Li 6708 $\AA$ feature, we used a spectral subtraction technique that required template spectra of similar effective temperature $\left(T_{\text {eff }}\right)$ and gravity $(\log g)$ to the targets (but without lithium) in order to isolate the contribution of Li. Templates were synthesized for $\log g=4.5$ at $100 \mathrm{~K}$ steps with a minimum of $4000 \mathrm{~K}$, using the MOOG spectral synthesis code (Sneden et al. 2012), with the (Kurucz 1992) solar-metallicity model atmospheres. Equivalent widths of the Li I $6708 \AA$ absorption line were measured by integrating under the relevant profile of the spectra after subtraction of the template. The extraction profile accounted for both the instrumental resolution, rotational broadening, and offset in RV. The line lists and atmosphere models do not include the strong molecular contributions that become important at low temperatures. For that reason, the lowest $T_{\text {eff }}$ used for the templates was $4000 \mathrm{~K}$, which leads to a systematic (but consistent) zero-point error in $\mathrm{EW}(\mathrm{Li})$ for stars cooler than this. However, this offset appears to be small (see Fig. 2), and these EWs are accurate enough to enable the selection of Li-rich objects (see Section 2.4). 


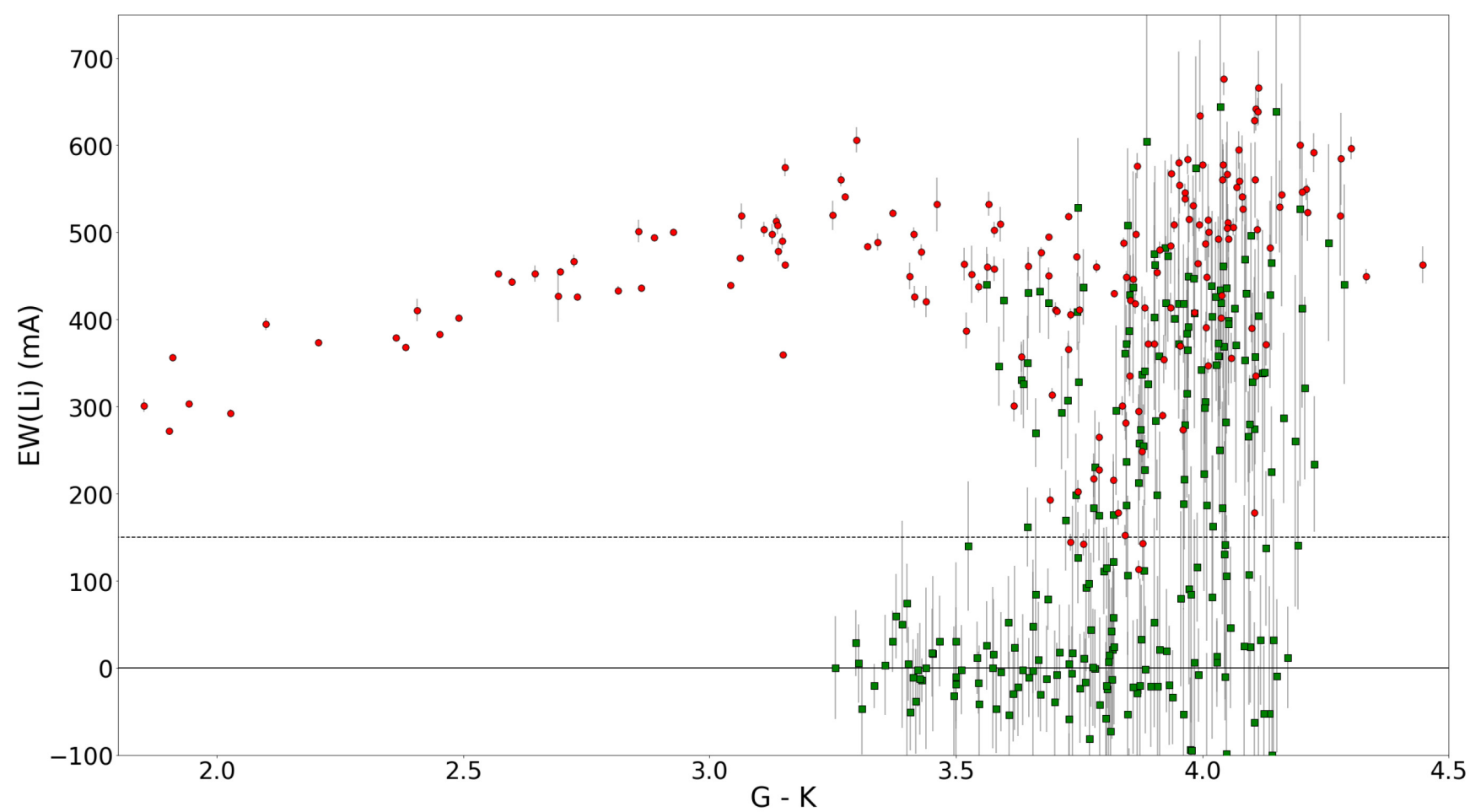

Figure 2. G-K colour versus EW(Li) for 248 AAT targets (green) with 'AAA' quality infrared photometry and EW(Li) measurements and 170 GES members (red) from Jeffries et al. (2014) with 'AAA' quality infrared photometry. The EW(Li) dividing line at $150 \mathrm{mÅ}$ is shown (see Section 2.4).

We obtained spectra for all 360 targets in this field, extracting RVs and $\mathrm{EW}(\mathrm{Li}) \mathrm{s}$ for 248 (68.9 per cent) of these with spectroscopy of sufficient quality (SNR $>5$ ). Of these, the median uncertainty in RV is $1.88 \mathrm{~km} \mathrm{~s}^{-1}$ and in $\mathrm{EW}(\mathrm{Li})$ is $80.26 \mathrm{m \AA}$. The $\mathrm{EW}(\mathrm{Li})$ for these 248 targets are shown in Fig. 2, compared with Li-rich members of $\gamma$ Vel defined in Jeffries et al. (2014).

\subsection{Compiling the sample}

We take the GES sample of the $\gamma$ Vel cluster (Jeffries et al. 2014) of 208 sources in a 0.9 square degree area and concatenate this with our AAT sample of 248 sources. Fifty-two sources have repeat observations in both GES and AAT samples and have measurements of $\mathrm{RV}$ and $\mathrm{EW}(\mathrm{Li})$ for both, so we calculated mean values weighted by the square of the inverse measurement uncertainty. We removed eight sources where RV measurements indicated these were binary systems and measured a median offset of $1.21 \mathrm{~km} \mathrm{~s}^{-1}$ between RV measurements for the remaining 44 sources. Since measurements from the GES sample are of higher quality than our AAT measurements, we add this median RV offset to all AAT RVs to bring the samples into agreement. At this stage, we have a sample of 422 unique sources with spectroscopic RVs and $\mathrm{EW}(\mathrm{Li})$.

On 2018 April 27, the second Gaia data release (DR2) became available, containing proper motion and parallax data for $\sim 97$ per cent of our sample. Twelve sources lack DR2 5-parameter astrometry, so we discard these. Sources were matched to the Gaia DR2 catalogue and then filtered on the suggested quality criteria to avoid using sources with spurious astrometric solutions (equations 1, 2, and 3 from Arenou et al. 2018). We also calculate renormalized unit weight error (RUWE) values for these sources (using Gaia DR2 RUWE data; see technical note GAIA-C3-TN-LU-LL-12401 ) and discard those with RUWE $>1.4$ as advised by Lindegren

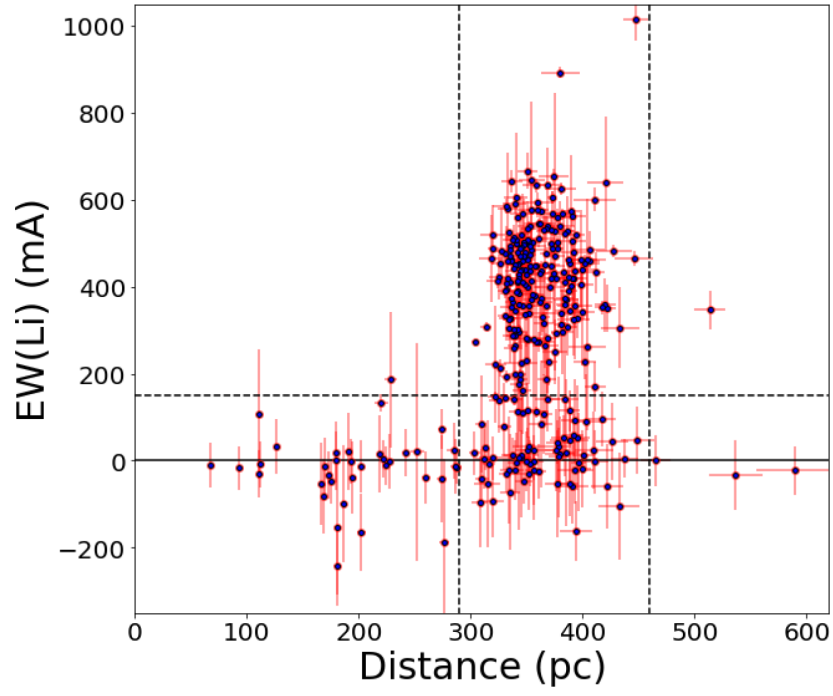

Figure 3. Distance estimates from Bailer-Jones et al. (2018) versus measured EW(Li) for 327 sources with distance $<600 \mathrm{pc}$ from the combined sample. The clear group between 290 and 460 pc with significant EW(Li)

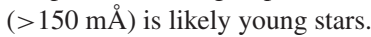

et al. (2018). Removing these leaves 339 unique sources with spectroscopic RVs, EW(Li), and 5-parameter astrometry.

\subsection{Selection of young stars}

Distance estimates were taken from Bailer-Jones et al. (2018) for all 341 sources with clean and complete spectroscopy and astrometry and these are shown in Fig. 3 for the 327 sources with distance $<600 \mathrm{pc}$, plotted against their $\mathrm{EW}(\mathrm{Li})$. There is 
a clear group apparent at 300-400 pc with significantly higher EW(Li) measurements than the rest of the sample that suggests these are young stars at the distance of the $\gamma$ Vel cluster. The distinction between the young stars and the contaminating field stars becomes unclear for EW(Li) $<150 \mathrm{~m} \AA$. In Jeffries et al. (2014), the criterion for GES sources to be considered young stars was $\mathrm{EW}(\mathrm{Li})>100 \mathrm{~m} \AA$, but since our AAT measurements have a lower precision than the GES data we set the threshold at $150 \mathrm{~m} \AA$ (see Figs 2 and 3). There could be a few highly Li-depleted objects that are filtered out from the sample at this stage, though they are likely $<10$ per cent of $\gamma$ Vel cluster members (Prisinzano et al. 2016; Jackson et al., in preparation).

\section{ANALYSIS}

\subsection{Member selection from the GES sample}

In Jeffries et al. (2014), the two populations were identified in their Li-rich sample using a two-component Gaussian fit to the $\mathrm{RV}$ distribution. For each source, membership probabilities $\left(P_{\mathrm{A}}\right.$, $P_{\mathrm{B}}$ ) were calculated from likelihood functions of the model RV distribution for the two populations. Sources were considered reasonably secure members of either population if either $P_{\mathrm{A}}$ or $P_{\mathrm{B}}>0.75$ (where $P_{\mathrm{A}}+P_{\mathrm{B}}=1$, i.e. the Li-rich sample was assumed uncontaminated).

\subsection{Member selection from the combined sample}

We use the Jeffries et al. (2014) membership information as a guide to help identify the differences in position and velocity between the two populations, and then define the boundaries using our new, larger sample.

Fig. 4 shows parallax versus RV for Li-rich [EW(Li) > $150 \mathrm{m \AA}]$ sources in our combined sample where sources from Jeffries et al. (2014) for population A are marked in red, population B in blue, and new sources from our observations in green. The separation between the high probability members of each population is clearly apparent in Fig. 4, and population A also occupies a smaller range in parallax. Based on the clustering apparent in Fig. 3 for sources with significant $\mathrm{EW}(\mathrm{Li})$, and on previous estimates of the distance of $\gamma^{2}$ Vel (e.g. $336_{-7}^{+8}$ pc; North et al. 2007) and Vela OB2 ( 410pc; de Zeeuw et al. 1999), we discard sources outside the parallax range $2.2<\varpi<3.45$ mas $(\sim 290-455 \mathrm{pc})$ as being unlikely to belong the Vela OB2 region. In Fig. 4, we define a box in parallax and RV around the population A (red) members, within which we select new sources as population A candidates. The edges are defined by $15.69<\mathrm{RV}<17.69 \mathrm{~km} \mathrm{~s}^{-1}$, which are the values $3 \sigma$ from the median RV of sources in population A (red), and $2.6<\varpi<3.2$ mas. We also require that for a source to be a member of population $\mathrm{A}$, it must lie within the circle in proper motion space illustrated in Fig. 5, of radius 0.6 mas yr $^{-1}$ centred on $\left(\mu_{\alpha}, \mu_{\delta}\right)=(-6.532,9.753)$ $\mathrm{km} \mathrm{s}^{-1}$. This selection circle was chosen as it includes the majority of population A members identified by Jeffries et al. (2014). Any other Li-rich target that is not located in both the parallax and RV box and proper motion circle is assigned to population $\mathrm{B}$.

After this selection process, we find 57 (26.4 per cent) sources consistent with being members of population A and 159 members of population B, in contrast to the results of Jeffries et al. (2014) who allocate 73 (52.5 per cent) sources to population A and 66 to population B. We end up with fewer sources in population A than Jeffries et al. (2014) due to imposing tighter restrictions on the membership of sources in population A from proper motion, and
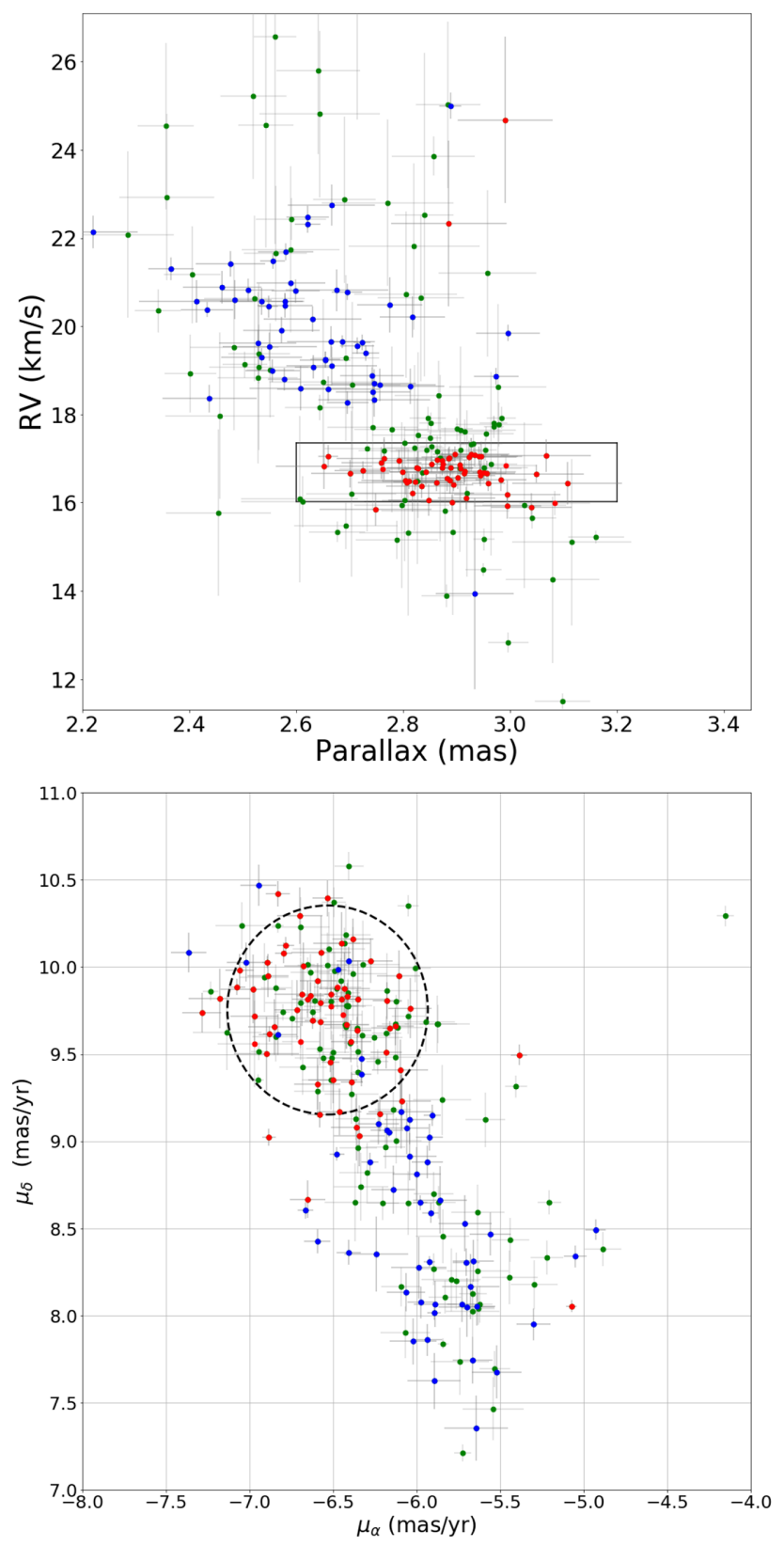

Figure 4. Top: Parallax versus RV of sources with $\mathrm{EW}(\mathrm{Li})>150 \mathrm{~m} \AA$ (green) from our spectroscopic survey along with population A (red) and B (blue) from Jeffries et al. (2014), showing the divisions we use to allocate sources to each population. Fifteen sources are beyond the RV range shown. Bottom: Gaia DR2 proper motions of the same sources.

not all of their original members are included in our final sample due to our Gaia DR2 astrometry cuts. In our final populations, 18 of the 159 population B members are GES sources that were allocated to population A by Jeffries et al. (2014), 40 of our population A members are Jeffries et al. (2014) population A members, and 53 of our population B members are Jeffries et al. (2014) population B members. The other 105 sources are new additions, 17 for population A and 88 for population $\mathrm{B}$.

Fig. 5 shows histograms of the proper motions, RV, and parallaxes of our final sample sources, with $3 \sigma$ outliers from the sample median removed, with population $\mathrm{A}$ members in red and population $\mathrm{B}$ members in blue. The clustered population A stands out as the peak 

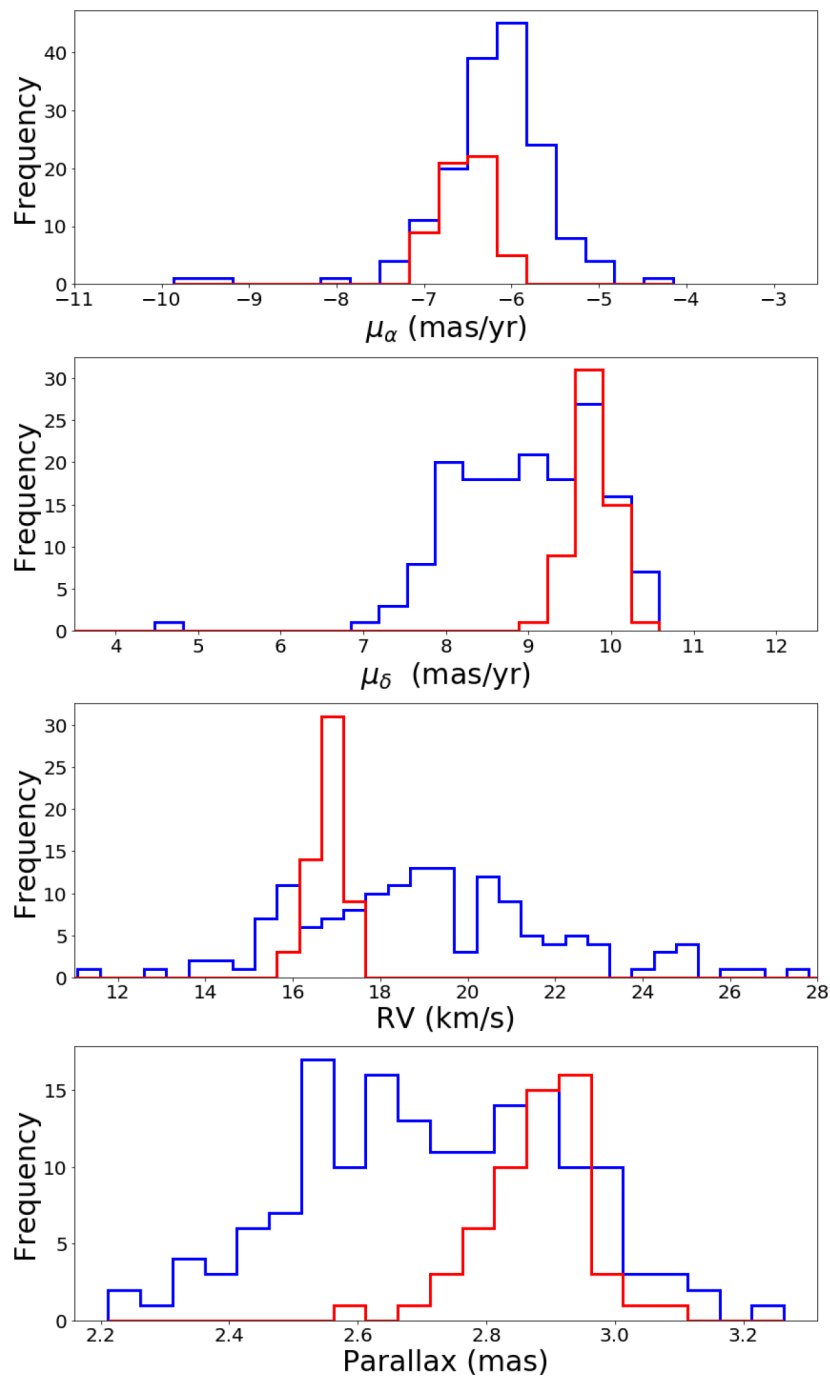

Figure 5. Histograms of the velocity and parallax data for sources from our sample with $\mathrm{EW}(\mathrm{Li})>150 \mathrm{~m} \AA$ without $3 \sigma$ outliers in any dimension that belong to populations A (red) and B (blue).

at $\mathrm{RV} \approx 17$ mas $\mathrm{yr}^{-1}$ and $\mu_{\delta} \approx 9.8$ mas $\mathrm{yr}^{-1}$, but the distinction is not clear in $\mu_{\alpha}$ or parallax where the two populations largely overlap.

Seven sources allocated to population A that lie outside the GES field are apparent in Fig. 6, though, due to the overlap of the two populations seen in Fig. 5, these may in fact be population $\mathrm{B}$ members. Otherwise, the majority of population A members are located within the original GES field, confirming the suggestion made by Jeffries et al. (2014) that this is a much more compact population than the widely spread population $\mathrm{B}$.

\subsection{Expansion trends}

Using Gaia DR2 positions, parallaxes, proper motions, and our combined sample of RVs, we estimate positions $\mathrm{X}, \mathrm{Y}, \mathrm{Z}$ and velocities $\mathrm{U}, \mathrm{V}, \mathrm{W}$ in the Galactic Cartesian coordinate system using a Bayesian inference method. This is done by forward modelling the observed equatorial coordinates, parallaxes, proper motions, and RVs from the modelled positions and velocities and the coordinate transformation matrices from Johnson \& Soderblom (1987). To

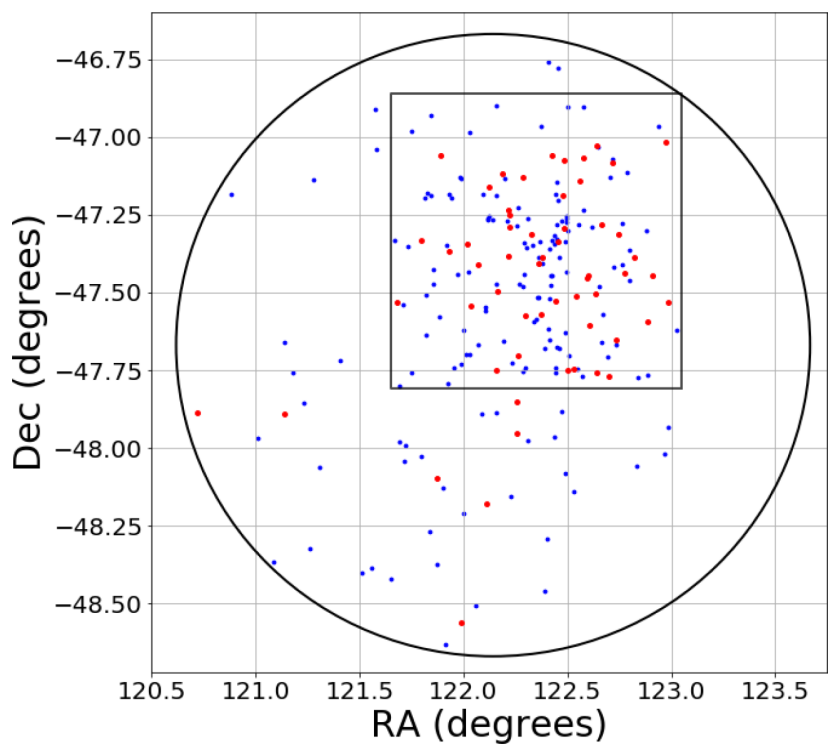

Figure 6. Positions of 57 population A (red) and 159 population B (blue) members from our final sample. The majority of population A members lie within the 0.9 degree square area observed by GES (Jeffries et al. 2014), but we also identify seven new population A members further south.

sample the posterior distribution function, we use the Markov Chain Monte Carlo (MCMC) sampler emcee (Foreman-Mackey et al. 2013). For each star, we perform 1000 iterations with 100 walkers in an unconstrained parameter space with flat and wide priors. We discard the first half of our iterations as a burn-in and from the second half we report the medians of the posterior distribution function as the best fit and use the 16th and 84th percentiles as the 1 sigma uncertainties. See Wright \& Mamajek (2018) for more details on this method.

This method is preferable to calculating $\mathrm{X}, \mathrm{Y}, \mathrm{Z}, \mathrm{U}, \mathrm{V}, \mathrm{W}$ from the measured quantities since measurement uncertainties are correlated and distance uncertainties, if derived from parallaxes, are not distributed as Gaussian (Bailer-Jones 2015).

In Fig. 7, we show positions X,Y,Z against velocities U,V,W for members of populations A and B in our sample. We calculate bestfitting linear relationships between these quantities using MCMC to fit linear relationships between position and velocity. The gradients and their uncertainties for each combination of position against velocity are given in Table 1 .

For $\mathrm{X}$ versus $\mathrm{U}$, $\mathrm{Y}$ versus $\mathrm{V}$, and $\mathrm{Z}$ versus $\mathrm{W}$, positive or negative gradients are an indication of expansion or contraction of the group (Blaauw 1964). We find evidence of expansion for population B of at least $4 \sigma$ significance in all three directions (gradients of $0.098_{-0.022}^{+0.021}, 0.044_{-0.007}^{+0.007}, 0.069_{-0.011}^{+0.011} \mathrm{~km} \mathrm{~s}^{-1} / \mathrm{pc}$ ), but this expansion is significantly anisotropic, the rate of expansion in the $\mathrm{X}$ direction being more than twice the rate in the $\mathrm{Y}$ direction. Using a twotailed $z$ test, we establish that the difference between the largest and smallest of these gradients is of at least $5 \sigma$ significance. We also find some evidence of expansion for population $\mathrm{A}$ in the $\mathrm{X}$ and $\mathrm{Z}$ directions $\left(0.091_{-0.044}^{+0.046}, 0.026_{-0.023}^{+0.022} \mathrm{~km} \mathrm{~s}^{-1} / \mathrm{pc}\right)$.

Due to $\gamma$ Vel's position in Galactic longitude $\left(\sim 263^{\circ}\right)$, the line of sight correlates closely to the Y direction, hence we would expect parallax uncertainty to contribute significantly to the estimation of $\mathrm{X}, \mathrm{Z}, \mathrm{U}$, and $\mathrm{W}$. This could create correlations between $\mathrm{X}$ and $\mathrm{U}$ values and $\mathrm{Z}$ and $\mathrm{W}$ values that would appear as signatures of expansion in those directions. We attempt to investigate the 

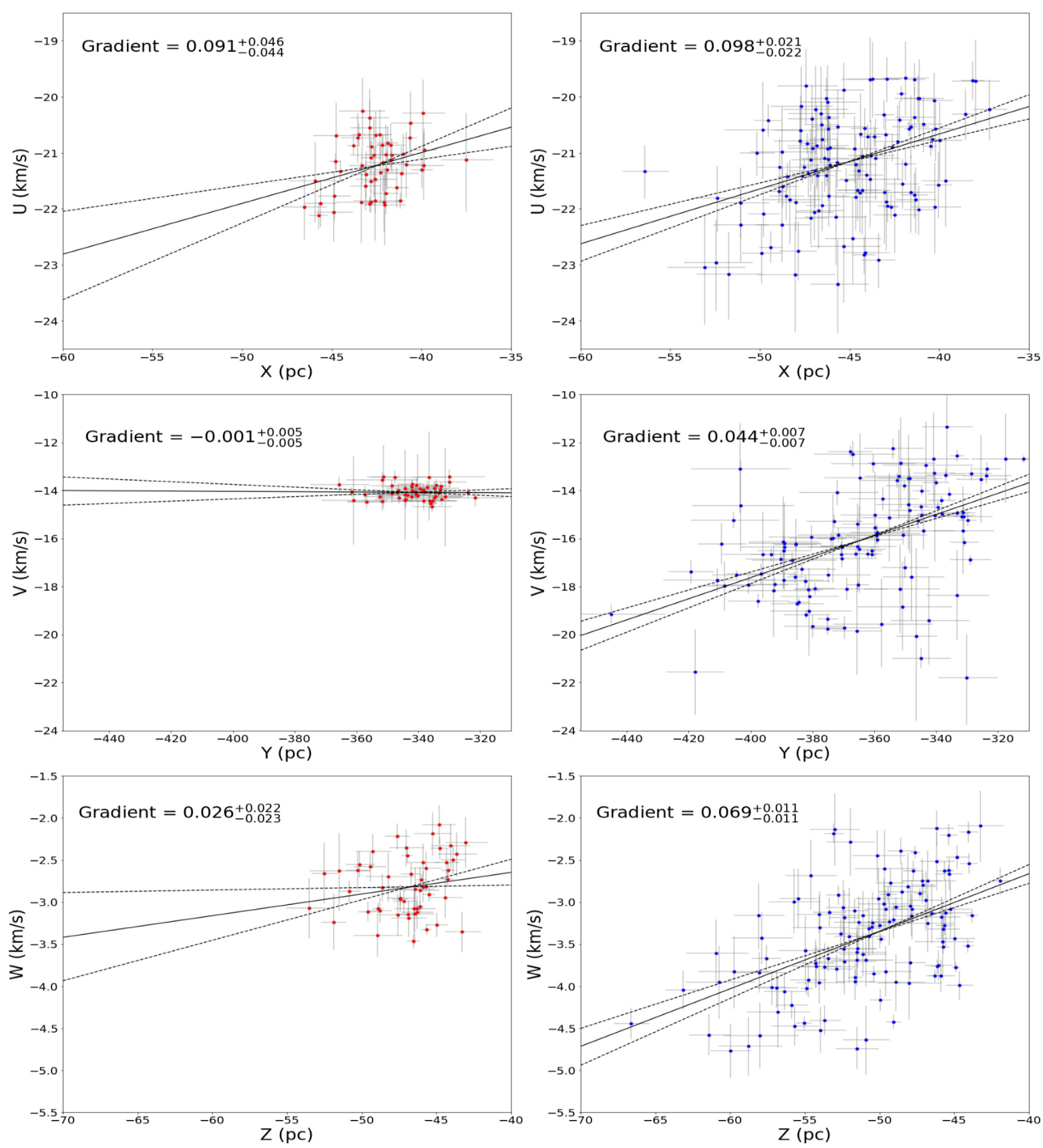

Figure 7. Cartesian position-velocity plots of populations A (red) and B (blue) with MCMC best-fitting correlation gradients and uncertainties plotted as solid and dashed lines centred on the mean values of each axis. Note that the ranges plotted in each row are different due to the different dispersions along each axis, but we kept the same range for plots along the same axes so the gradients can be compared.

effect of this covariance in our Cartesian positions and velocities by generating a sample of 1000 stars with Gaussian X,Y,Z,U,V,W distributions defined by the mean and standard deviations of these values for our population A members. We use the coordinate transformation matrices from Johnson \& Soderblom (1987) to calculate positions, parallaxes, proper motions, and RVs for this sample and then add random parallax, proper motion, and RV uncertainties from Gaussian distributions with the standard deviations of these uncertainty values for our population A members. We then calculate Cartesian positions and velocities by inverting the previous coordinate transformation matrices. We find, in fact, that the contributions to the position-velocity correlations from correlated uncertainties are small in comparison to our measured gradients $\left(<0.01 \mathrm{~km} \mathrm{~s}^{-1} / \mathrm{pc}\right)$ and do not change the significance levels of the expansion signatures.

\subsection{Cluster rotation}

Rotation is evidenced by correlations between positions $\mathrm{X}, \mathrm{Y}, \mathrm{Z}$ and velocities $\mathrm{U}, \mathrm{V}, \mathrm{W}$ in different directions. There is some evidence for rotation in population A in several dimensions (see Table 1), but the most significant signature is found in $\mathrm{Y}$ versus $\mathrm{U}$ at $3 \sigma$ significance $\left(0.029_{-0.009}^{+0.008} \mathrm{~km} \mathrm{~s}^{-1} / \mathrm{pc}\right.$; Fig. 8). However, interpreting signatures of rotation is more complex than linear expansion or contraction: the 
Table 1. Gradients of MCMC linear best-fitting models for both A and B populations of the $\gamma$ Vel cluster for every combination of Cartesian position and velocity dimensions, as well as uncertainties given by the 16th and 84th percentiles of MCMC fits.

\begin{tabular}{lcrc}
\hline Velocity & Position & $\begin{array}{r}\text { Pop. A gradient } \\
\left(\mathrm{km} \mathrm{s}^{-1} / \mathrm{pc}\right)\end{array}$ & $\begin{array}{c}\text { Pop. B gradient } \\
\left(\mathrm{km} \mathrm{s}^{-1} / \mathrm{pc}\right)\end{array}$ \\
\hline $\mathrm{U}$ & $\mathrm{X}$ & $0.091_{-0.044}^{+0.046}$ & $0.098_{-0.022}^{+0.021}$ \\
$\mathrm{~V}$ & $\mathrm{X}$ & $-0.027_{-0.026}^{+0.025}$ & $0.298_{-0.052}^{+0.051}$ \\
$\mathrm{~W}$ & $\mathrm{X}$ & $0.037_{-0.020}^{+0.029}$ & $0.084_{-0.015}^{+0.015}$ \\
$\mathrm{U}$ & $\mathrm{Y}$ & $0.029_{-0.009}^{+0.000}$ & $0.009_{-0.003}^{+0.003}$ \\
$\mathrm{~V}$ & $\mathrm{Y}$ & $-0.001_{-0.005}^{+0.005}$ & $0.044_{-0.007}^{+0.007}$ \\
$\mathrm{~W}$ & $\mathrm{Y}$ & $0.002_{-0.005}^{+0.006}$ & $0.014_{-0.002}^{+0.002}$ \\
$\mathrm{U}$ & $\mathrm{Z}$ & $0.030_{-0.033}^{+0.033}$ & $0.033_{-0.016}^{+0.015}$ \\
$\mathrm{~V}$ & $\mathrm{Z}$ & $0.003_{-0.022}^{+0.023}$ & $0.230_{-0.037}^{+0.037}$ \\
$\mathrm{~W}$ & $\mathrm{Z}$ & $0.026_{-0.023}^{+0.023}$ & $0.069_{-0.011}^{+0.011}$ \\
\hline
\end{tabular}

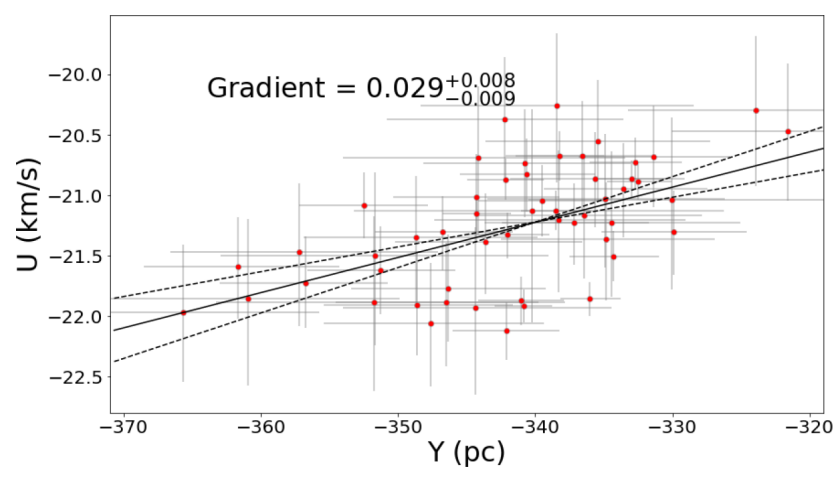

Figure 8. Cartesian position Y-velocity U of population A with MCMC best-fitting correlation gradient and uncertainty plotted as solid and dashed lines centred on the mean values of each axis. The significant $(3 \sigma)$ positive gradient is strong evidence of rotation in this direction.

same motion may have signatures in multiple dimensions depending on the orientation of the axis of rotation, so we are hesitant to draw physical conclusions from this. Rotation in bound clusters has been observed previously but not frequently. In Hénault-Brunet et al. (2012), evidence for rotation was discovered in the cluster $\mathrm{R} 136$, and it was argued that clusters may form with at least $\sim 20$ per cent of their kinetic energy in rotation. It will be difficult to put a precise angular velocity to the $\gamma$ Vel cluster without further data and modelling.

\section{DISCUSSION}

The results of the previous section strengthen the hypothesis that population A belongs to the $\gamma$ Vel cluster and that population $\mathrm{B}$ belongs to the wider Vela OB2 association, and have interesting implications for the possible formation and evolution mechanisms of these groups.

If population B is indeed part of the wider Vela OB2 association, the expansion trends in each dimension would be expected following residual gas expulsion. According to some models (e.g. Baumgardt \& Kroupa 2007), such expansion trends are expected to be isotropic, but the velocity gradients of this group are, in fact, strongly anisotropic. However, more recent studies (e.g. Kruijssen et al. 2012) suggest that residual gas expulsion may not necessarily produce isotropic expansion patterns and therefore more theoretical work exploring the predicted expansion patterns due to residual gas expulsion is needed. Numerical simulations of residual gas expulsion will be needed to determine whether this mechanism can produce the kinematic behaviour we have found.

Such strong evidence for expansion in an association is by no means commonplace. Other recent studies using Gaia astrometry have not found evidence for expansion in other associations (e.g. Wright et al. 2016; Ward \& Kruijssen 2018; Wright \& Mamajek 2018). Cantat-Gaudin et al. (2019a) also studied the Vela complex and identified signatures of anisotropic expansion in many of the populations present there. Unlike the previously mentioned studies, Cantat-Gaudin et al. (2019a) used the unsupervized classification scheme UPMASK to differentiate between multiple populations in their sample differing in position, proper motion, and parallax. Likewise, we have used the two-component model of the $\gamma$ Vel population from Jeffries et al. (2014) to separate two kinematically distinct populations in our sample. The results from these studies may indicate a need to distinguish subgroups present in associations in order to detect the kinematic signatures of expansion that exist.

If we instead treat our sample as one group, rather than dividing it into two populations, we still find significant signatures for expansion in each dimension, as we identified for population B.

\section{SUMMARY}

We have selected a sample of likely PMS stars in a 2-degree diameter area in the vicinity of the $\gamma$ Vel cluster using Gaia photometry and obtained spectroscopic RVs and EW(Li) measurements for 248 of them. We combine these with the GES $\gamma$ Vel field sample (Jeffries et al. 2014) located within the area of our new observations and with Gaia DR2. We separate the sample into the two populations identified by Jeffries et al. (2014) using RVs, proper motions, and parallaxes. Seven population A members lie outside the GES field, but the majority of population A is located within the smaller GES field, while population B is spread across the whole field.

We find significant signatures of expansion for population B in all three dimensions, which fits with the idea that this population is part of the wider, unbound Vela OB2 association that is in the process of expanding. The rates of expansion in each dimension are also found to be significantly asymmetric.

For population A there is no significant signature of expansion in $\mathrm{Y}$ or $\mathrm{Z}$ directions, which fits with this population belonging to a potentially bound $\gamma$ Vel cluster, though there is a signature of expansion in the $\mathrm{X}$ direction. There is some evidence for rotation, with the most significant signature present in $\mathrm{Y}$ versus $\mathrm{U}$.

In order to determine the likely evolution scenario responsible for the asymmetric expansion we have found in this study, and to identify kinematic signatures in stellar populations across the wider area of the Vela complex, a large-scale spectroscopic survey over the area of the Vela OB2 association will be necessary to confirm youth and combine with Gaia to give 6D kinematics.

\section{ACKNOWLEDGEMENTS}

We thank the referee for their time and a helpful referee's report that has improved this paper. NJW acknowledges an STFC Ernest Rutherford Fellowship (grant number ST/M005569/1). This work has made use of data from the European Space Agency (ESA) space mission Gaia, processed by the Gaia Data Processing and Analysis Consortium (DPAC). Funding for DPAC has been provided by national institutions, in particular the institutions participating in the Gaia Multilateral Agreement. This research made use of the Simbad 
and VizieR catalogue access tools (provided by CDS, Strasbourg, France), Astropy for PYTHON (Astropy Collaboration et al. 2013) and TOPCAT (Taylor 2005).

\section{REFERENCES}

AAO Software Team, 2015, 2dfdr: Data reduction software

Ambartsumian V., 1947, Évolyutsiya zvezd i astrofizika. Izdatel'stvo Akad Nauk Arm SSR, Erevan

Arenou F. et al., 2018, A\&A, 616, A17

Armstrong J. J., Wright N. J., Jeffries R. D., 2018, MNRAS, 480, L121

Astropy Collaboration et al., 2013, A\&A, 558, A33

Bailer-Jones C. A. L., 2015, PASP, 127, 994

Bailer-Jones C. A. L., Rybizki J., Fouesneau M., Mantelet G., Andrae R., 2018, AJ, 156, 58

Baumgardt H., Kroupa P., 2007, MNRAS, 380, 1589

Beccari G., Boffin H. M. J., Jerabkova T., Wright N. J., Kalari V. M., Carraro G., De Marchi G., de Wit W.-J., 2018, MNRAS, 481, L11

Blaauw A., 1964, ARA\&A, 2, 213

Briceño C., Hartmann L., Hernández J., Calvet N., Vivas A. K., Furesz G., Szentgyorgyi A., 2007, ApJ, 661, 1119

Cantat-Gaudin T., Mapelli M., Balaguer-Núñez L., Jordi C., Sacco G., Vallenari A., 2019b, A\&A, 621, A115

Cantat-Gaudin T. et al., 2019a, A\&A, 626, A17

Damiani F., Prisinzano L., Jeffries R. D., Sacco G. G., Randich S., Micela G., 2017, A\&A, 602, L1

de Zeeuw P. T., Hoogerwerf R., de Bruijne J. H. J., Brown A. G. A., Blaauw A., 1999, AJ, 117, 354

Ester M., Kriegel H.-P., Sander J., Xu X., 1996, KDD, 96, 226

Foreman-Mackey D., Hogg D. W., Lang D., Goodman J., 2013, PASP, 125 , 306

Franciosini E., Sacco G. G., Jeffries R. D., Damiani F., Roccatagliata V., Fedele D., Randich S., 2018, A\&A, 616, L12

Gaia Collaboration, 2018, A\&A, 616, A1

Gaia Collaboration et al., 2016, A\&A, 595, A1

Gilmore G. et al., 2012, The Messenger, 147, 25

Hills J. G., 1980, ApJ, 235, 986

Hénault-Brunet V. et al., 2012, A\&A, 545, L1

Jackson R. J., Deliyannis C. P., Jeffries R. D., 2018, MNRAS, 476, 3245

Jackson R. J., Jeffries R. D., 2010, MNRAS, 407, 465

Jeffries R. D., Naylor T., Walter F. M., Pozzo M. P., Devey C. R., 2009, MNRAS, 393, 538

Jeffries R. D. et al., 2014, A\&A, 563, A94
Jeffries R. D. et al., 2017, MNRAS, 464, 1456

Johnson D. R. H., Soderblom D. R., 1987, AJ, 93, 864

Krone-Martins A., Moitinho A., 2014, A\&A, 561, A57

Kroupa P., Aarseth S., Hurley J., 2001, MNRAS, 321, 699

Kruijssen J. M. D., 2012, MNRAS, 426, 3008

Kruijssen J. M. D., Maschberger T., Moeckel N., Clarke C. J., Bastian N., Bonnell I. A., 2012, MNRAS, 419, 841

Kuhn M. A., Hillenbrand L. A., Sills A., Feigelson E. D., Getman K. V., 2019, ApJ, 870, 32

Kurucz R. L., 1992, in Barbuy B., Renzini A., eds, IAU Symposium, Vol. 149, The Stellar Populations of Galaxies, Kluwer Academic Publishers, Dordrecht. p. 225

Lewis I. J. et al., 2002, MNRAS, 333, 279

Lindegren L. et al., 2018, A\&A, 616, A2

McMahon R. G., Banerji M., Gonzalez E., Koposov S. E., Bejar V. J., Lodieu N., Rebolo R., VHS Collaboration, 2013, The Messenger, 154, 35

North J. R., Tuthill P. G., Tango W. J., Davis J., 2007, MNRAS, 377, 415

Pecaut M. J., Mamajek E. E., 2016, MNRAS, 461, 794

Pozzo M., Jeffries R. D., Naylor T., Totten E. J., Harmer S., Kenyon M., 2000, MNRAS, 313, L23

Preibisch T., Brown A. G. A., Bridges T., Guenther E., Zinnecker H., 2002, AJ, 124, 404

Prisinzano L. et al., 2016, A\&A, 589, A70

Randich S., Gilmore G., Gaia-ESO Consortium, 2013, The Messenger, 154, 47

Sacco G. G. et al., 2015, A\&A, 574, L7

Sheinis A. et al., 2015, J. Astron. Telesc., Instrum., and Syst., 1, 035002

Skrutskie M. F. et al., 2006, AJ, 131, 1163

Sneden C., Bean J., Ivans I., Lucatello S., Sobeck J., 2012, MOOG: LTE line analysis and spectrum synthesis

Taylor M. B., 2005, in Shopbell P., Britton M., Ebert R., eds, ASP Conf. Ser., Vol. 347, Astronomical Data Analysis Software and Systems XIV. Astron. Soc. Pac., San Francisco. p. 29

Tutukov A. V., 1978, A\&A, 70, 57

Ward J. L., Kruijssen J. M. D., 2018, MNRAS, 475, 5659

Wright N. J., Bouy H., Drew J. E., Sarro L. M., Bertin E., Cuillandre J.-C., Barrado D., 2016, MNRAS, 460, 2593

Wright N. J., Mamajek E. E., 2018, MNRAS, 476, 381

Wright N. J. et al., 2019, MNRAS, 486, 2477

This paper has been typeset from a $\mathrm{T}_{\mathrm{E}} \mathrm{X} / \mathrm{LAT} \mathrm{E}$ file prepared by the author. 